\title{
The compact support property for measure-valued processes
}

\author{
János Engländer ${ }^{\mathrm{a}}$, Ross G. Pinsky ${ }^{\mathrm{b}, *}$ \\ a Department of Statistics and Applied Probability, University of California, Santa Barbara, CA 93106-3110, USA \\ ${ }^{\mathrm{b}}$ Department of Mathematics Technion-Israel Institute of Technology, Haifa, 32000, Israel
}

Received 21 December 2004; received in revised form 14 April 2005; accepted 11 July 2005

Available online 9 December 2005

\begin{abstract}
The purpose of this article is to give a rather thorough understanding of the compact support property for measure-valued processes corresponding to semi-linear equations of the form

$$
\begin{aligned}
& u_{t}=L u+\beta u-\alpha u^{p} \quad \text { in } \mathbb{R}^{d} \times(0, \infty), p \in(1,2] ; \\
& u(x, 0)=f(x) \quad \text { in } \mathbb{R}^{d} ; \\
& u(x, t) \geqslant 0 \quad \text { in } \mathbb{R}^{d} \times[0, \infty) .
\end{aligned}
$$

In particular, we shall investigate how the interplay between the underlying motion (the diffusion process corresponding to $L$ ) and the branching affects the compact support property. In [J. Engländer, R. Pinsky, On the construction and support properties of measure-valued diffusions on $D \subset \mathbb{R}^{d}$ with spatially dependent branching, Ann. Probab. 27 (1999) 684-730], the compact support property was shown to be equivalent to a certain analytic criterion concerning uniqueness of the Cauchy problem for the semi-linear parabolic equation related to the measured valued process. In a subsequent paper [J. Englan̈der, R. Pinsky, Uniqueness/nonuniqueness for nonnegative solutions of second-order parabolic equations of the form $u_{t}=L u+V u-\gamma u^{p}$ in $\mathbb{R}^{n}$, J. Differential Equations 192 (2003) 396-428], this analytic property was investigated purely from the point of view of partial differential equations. Some of the results obtained in this latter paper yield interesting results concerning the compact support property. In this paper, the results from [J. Englainder, R. Pinsky, Uniqueness/nonuniqueness for nonnegative solutions of secondorder parabolic equations of the form $u_{t}=L u+V u-\gamma u^{p}$ in $\mathbb{R}^{n}$, J. Differential Equations 192 (2003) 396-428] that are relevant to the compact support property are presented, sometimes with extensions. These results are interwoven with new results and some informal heuristics. Taken together, they yield a rather comprehensive picture of the compact support property. Inter alia, we show that the concept of a measure-valued process hitting a point can be investigated via the compact support property, and suggest an alternate proof of a result concerning the hitting of points by super-Brownian motion.
\end{abstract}

(c) 2005 Elsevier SAS. All rights reserved.

\section{Résumé}

Le but de cet article est de donner une assez pleine compréhension de la propriété du support compact pour les processus à valeurs mesures qui correspondent aux équations semi-linéaires de la forme

\footnotetext{
* Corresponding author.

E-mail addresses: englander@ pstat.ucsb.edu (J. Engländer), pinsky@ math.technion.ac.il (R.G. Pinsky).

URLs: http://www.pstat.ucsb.edu/faculty/englander, http://www.math.technion.ac.il/people/pinsky/.
} 


$$
\begin{aligned}
& u_{t}=L u+\beta u-\alpha u^{p} \quad \text { in } \mathbb{R}^{d} \times(0, \infty), p \in(1,2] ; \\
& u(x, 0)=f(x) \quad \text { in } \mathbb{R}^{d} ; \\
& u(x, t) \geqslant 0 \quad \text { in } \mathbb{R}^{d} \times[0, \infty) .
\end{aligned}
$$

En particulier, nous recherchons comment la relation entre le processus de diffusion correspondant à $L$ et le branchement peut affecter la propriété du support compact. En [J. Engländer, R. Pinsky, On the construction and support properties of measurevalued diffusions on $D \subset \mathbb{R}^{d}$ with spatially dependent branching, Ann. Probab. 27 (1999) 684-730], la propriété du support compact était montrée être équivalente à un certain critère analytique qui concerne l'unicité du probléme de Cauchy pour l'équation semilineaire parabolique correspondant au processus à valeurs mesures. Dans un article subséquent [J. Engländer, R. Pinsky, Uniqueness/nonuniqueness for nonnegative solutions of second-order parabolic equations of the form $u_{t}=L u+V u-\gamma u^{p}$ in $\mathbb{R}^{n}$, J. Differential Equations 192 (2003) 396-428], cette propriété analytique était étudiée du point de vue des équations aux dérivées partielles. Quelques uns des résultats obtenus dans ce dernier papier fournissent des résultats intéressants sur la propriété du support compact. Dans cet article, les résultats de [J. Engländer, R. Pinsky, Uniqueness/nonuniqueness for nonnegative solutions of second-order parabolic equations of the form $u_{t}=L u+V u-\gamma u^{p}$ in $\mathbb{R}^{n}$, J. Differential Equations 192 (2003) 396-428] qui sont applicables à la propriété du support sont présentés, parfois avec des extensions. Ces résultats sont mêlés à de nouveaux résultats et à des heuristiques. Dans l'ensemble, ils donnent un tableau assez clair de la propriété du support compact. Parmi les autres résultats, nous montrons que le concept du processus à valeurs mesures atteignant un point peut être étudié via la propriété du support compact, et nous suggérons une preuve différente pour un résultat concernant l'atteinte des points par le super-mouvement brownien.

(c) 2005 Elsevier SAS. All rights reserved.

MSC: primary: 60J60, 60J80; secondary: 35K15, 35K55

Keywords: Semilinear equation; Elliptic equation; Positive solutions; Uniqueness of the Cauchy problem; Compact support property;

Superprocess; Superdiffusion; Super-Brownian motion; Measure-valued process; $h$-transform; Weighted superprocess

\section{Introduction and statement of results}

The purpose of this article is to give a rather thorough understanding of the compact support property for measurevalued diffusion processes. In particular, we shall investigate how the interplay between the underlying motion and the branching affects the compact support property. In [10], the compact support property was shown to be equivalent to a certain analytic criterion concerning uniqueness of the Cauchy problem for the semi-linear parabolic equation related to the measured valued process. In a subsequent paper [11], this analytic property was investigated purely from the point of view of partial differential equations. Some of the results obtained in this latter paper yield interesting results concerning the compact support property. In this paper, the results from [11] that are relevant to the compact support property are presented, sometimes with extensions. These results are interwoven with new results and some informal heuristics. Taken together, they yield a rather comprehensive picture of the compact support property. Inter alia, we show that the concept of a measure-valued process hitting a point can be investigated via the compact support property and suggest an alternate proof of a result concerning the hitting of points by super-Brownian motion.

We state all of our results for the case that the underlying space is $\mathbb{R}^{d}$; however, all the results also hold for generic domains in $\mathbb{R}^{d}$ - see [10], where the superprocesses studied in this paper are constructed on generic Euclidean domains.

We begin by defining the measure-valued processes under study. Let

$$
L=\frac{1}{2} \sum_{i, j=1}^{d} a_{i, j} \frac{\partial^{2}}{\partial x_{i} \partial x_{j}}+\sum_{i=1}^{d} b_{i} \frac{\partial}{\partial x_{i}},
$$

where $a_{i, j}, b_{i} \in C^{\alpha}\left(\mathbb{R}^{d}\right)$, with $\alpha \in(0,1)$, and $\left\{a_{i, j}\right\}$ is strictly elliptic; that is, $\sum_{i, j=1}^{d} a_{i, j}(x) v_{i} v_{j}>0$, for all $x \in \mathbb{R}^{d}$ and $v \in \mathbb{R}^{d}-\{0\}$. Let $Y(t)$ denote the diffusion process corresponding to the generalized martingale problem for $L$ on $\mathbb{R}^{d}$ [14], and denote corresponding probabilities by $\left\{\mathcal{P}_{x} ; x \in \mathbb{R}^{d}\right\}$. Denote the lifetime of $Y(t)$ by $\tau_{\infty}$. One has $\tau_{\infty}=\lim _{n \rightarrow \infty} \tau_{n}$, where $\tau_{n}=\inf \{t \geqslant 0:|Y(t)| \geqslant n\}$. Recall that $Y(t)$ is called non-explosive (or conservative) if $\mathcal{P}_{x}\left(\tau_{\infty}<\infty\right)=0$, for some, or equivalently all, $x \in \mathbb{R}^{d}$; otherwise $Y(t)$ is called explosive. The process $Y(t)$ serves as the underlying motion of the measure-valued process. 
The branching mechanism is of the form $\Phi(x, z)=\beta(x) z-\alpha(x) z^{p}$, where $p \in(1,2], \beta$ is bounded from above, $\alpha>0$, and $\alpha, \beta \in C^{\kappa}\left(\mathbb{R}^{d}\right)$, for some $\kappa \in(0,1]$. A finite measure-valued process $X(t)=X(t, \cdot)$ is the Markov process defined uniquely via the following log-Laplace equation:

$$
E(\exp (-\langle f, X(t)\rangle) \mid X(0)=\mu)=\exp \left(-\left\langle u_{f}(\cdot, t), \mu\right\rangle\right)
$$

for $f \in C_{c}^{+}\left(\mathbb{R}^{d}\right)$, the space of compactly supported, nonnegative, continuous functions on $\mathbb{R}^{d}$, and for finite initial measures $\mu$, where $u_{f}$ is the minimal positive solution to the evolution equation

$$
\begin{aligned}
& u_{t}=L u+\beta u-\alpha u^{p} \quad \text { in } \mathbb{R}^{d} \times(0, \infty) ; \\
& u(x, 0)=f(x) \quad \text { in } \mathbb{R}^{d} ; \\
& u(x, t) \geqslant 0 \quad \text { in } \mathbb{R}^{d} \times[0, \infty) .
\end{aligned}
$$

(For the construction, see [10].) The measure for the process started from $\mu$ will be denoted by $P_{\mu}$, and its expectation operator will be denoted by $E_{\mu}$.

We recall the compact support property.

Definition. Let $\mu \in \mathcal{M}_{F}\left(\mathbb{R}^{d}\right)$ be compactly supported. The measure-valued process corresponding to $P_{\mu}$ possesses the compact support property if

$$
P_{\mu}\left(\bigcup_{0 \leqslant s \leqslant t} \operatorname{supp} X(s) \text { is bounded }\right)=1, \quad \text { for all } t \geqslant 0 .
$$

Remark. The parameter $\beta$ may be thought of as the mass creation parameter (see the discussion of the particle process approximation to the measure-valued process at the end of this section). It is possible to extend the construction of the measure-valued process to certain $\beta$ which are unbounded from above; namely, to those $\beta$ for which the generalized principal eigenvalue of the operator $L+\beta$ is finite. However, the resulting process has paths which are mutually absolutely continuous with respect to another measure-valued process whose mass creation parameter $\beta$ is bounded from above [10]. Thus, the compact support property will hold for the former process if and only if it holds for the latter one. Without further mention, it will always be assumed in this paper that $\beta$ is bounded from above.

There are four objects, corresponding to four different underlying probabilistic effects, which can influence the compact support property:

(1) $L$, the operator corresponding to the underlying motion;

(2) $\beta$, the mass creation parameter of the branching mechanism;

(3) $\alpha$, the nonlinear component of the branching mechanism, which can be thought of as the variance parameter if $p=2$;

(4) $p$, the power of the nonlinearity, which is the scaling power and is connected to the fractional moments of the offspring distribution in the particle process approximation to the measure-valued process.

(For (2), (3) and (4) above, see the discussion of the particle process approximation to the measure-valued process at the end of this section.)

We shall see that both $L$ and $\alpha$ play a large role in determining whether or not the compact support property holds; $\beta$ and $p$ play only a minor role.

In [10], the compact support property was shown to be equivalent to a uniqueness property for solutions to (1.2).

Theorem EP1. The compact support property holds for one, or equivalently all, nonzero, compactly supported initial measures $\mu$ if and only if there are no nontrivial solutions to (1.2) with initial data $f \equiv 0$.

Remark 1. We emphasize that the uniqueness property in Theorem EP1 concerns all classical solutions to (1.2), with no growth restrictions. If one restricts to mild solutions-solutions which solve an integral equation involving the linear semigroup corresponding to the operator $L$-then, for example, uniqueness holds in this class if $\alpha, \beta$ and 
the coefficients of $L$ are bounded [13]; yet, these conditions certainly do not guarantee uniqueness in the class of all positive, classical solutions (see Theorem 1 below).

Remark 2. In fact, the proof of Theorem EP1 shows that there exists a maximal solution $u_{\max }$ to (1.2) with initial data $f=0$, and

$$
P_{\mu}\left(\bigcup_{0 \leqslant s \leqslant t} \operatorname{supp} X(s) \text { is bounded }\right)=\exp \left(-\int_{\mathbb{R}^{d}} u_{\max }(x, t) \mu(\mathrm{d} x)\right),
$$

for compactly supported $\mu$. This shows that when the compact support property fails, the onset of the failure is gradual; that is, as a function of $t, P_{\mu}\left(\bigcup_{0 \leqslant s \leqslant t} \operatorname{supp} X(s)\right.$ is bounded) is continuous and equal to 1 at time $t=0$. This behavior is in contrast to the behavior of the measure-valued process corresponding to the semi-linear operator $u_{t}=\Delta u-u \log u$, investigated recently in [12]. This process is obtained as a weak limit as $p \rightarrow 1$ of the processes corresponding to the semi-linear operators

$$
u_{t}=\Delta u+\frac{1}{p-1} u-\frac{1}{p-1} u^{p} .
$$

Unlike the measure-valued processes defined above, this process is immortal; that is, $P_{\mu}(X(t)=0)=0$, for all $t \geqslant 0$. Furthermore, it is shown that $P_{\mu}\left(\bigcup_{0 \leqslant s \leqslant t} \operatorname{supp} X(s)\right.$ is bounded $)=0$, for all $t>0$. Thus, the onset of the failure of the compact support property is instantaneous. Theorem EP1 is not valid for this process. Indeed, the proof of Theorem EP1 requires the fact that a maximal solution exists for (1.2) with initial condition $f=0$. The existence of such a maximal solution is essentially equivalent to the existence of a universal, a priori upper bound on all solutions to (1.2); that is, the existence of a finite function $M(x, t)$ on $\mathbb{R}^{d} \times(0, \infty)$ such that $u(x, t) \leqslant M(x, t)$, for all $(x, t) \in$ $\mathbb{R}^{d} \times(0, \infty)$, and all solutions $u$ to (1.2). Such a universal a priori upper bound does not exist for the equation

$$
u_{t}=\Delta u-u \log u \text {. }
$$

In [15], a more or less necessary and sufficient condition on the nonlinear term (independent of the operator $L$ ) is given for the existence of such a bound.

Remark 3. Theorem EP1 suggests a parallel between the compact support property for measure-valued processes and the non-explosion property for diffusion processes. Indeed, the non-explosion property for the diffusion process $Y(t)$ corresponding to the operator $L$ is equivalent to the nonexistence of nontrivial, bounded, positive solutions to the linear Cauchy problem with 0 initial data:

$$
\begin{aligned}
& u_{t}=L u \quad \text { in } \mathbb{R}^{d} \times(0, \infty) ; \\
& u(x, 0)=0 \quad \text { in } \mathbb{R}^{d} ; \\
& u \geqslant 0 \quad \text { in } \mathbb{R}^{d} \times(0, \infty) .
\end{aligned}
$$

(For one direction of this result, note that $u(x, t) \equiv \mathcal{P}_{x}\left(\tau_{\infty} \leqslant t\right)$ serves as a nontrivial solution to (1.4) in the explosive case.) It is natural for bounded, positive solutions to be the relevant class of solutions in the linear case and for positive solutions to be the relevant class of solutions in the semi-linear case. Indeed, by Ito's formula, the probabilities for certain events related to $Y(t)$ are obtained as bounded, positive solutions to the linear equation, and by the log-Laplace equation, the negative of the logarithm of the probability of certain events related to $X(t)$ can be obtained as positive solutions to the semi-linear equation.

The class of operators $L$ satisfying the following assumption will play an important role.

Assumption 1. For some $C>0$,

(1) $\sum_{i, j=1}^{n} a_{i j}(x) v_{i} v_{j} \leqslant C|v|^{2}\left(1+|x|^{2}\right), x, v \in \mathbb{R}^{d}$;

(2) $|b(x)| \leqslant C(1+|x|), x \in \mathbb{R}^{d}$.

The next theorem culls some results from [11] and applies them to the probabilistic setting at hand. 
Theorem EP2. Let $p \in(1,2]$ and let the coefficients of L satisfy Assumption 1.

(1) There is no nontrivial solution to (1.4); thus, the diffusion process $Y(t)$ does not explode.

(2) If

$$
\inf _{x \in \mathbb{R}^{d}} \alpha(x)>0,
$$

then there is no nontrivial solution to (1.2) with initial data $f=0$; thus, the compact support property holds for $X(t)$.

Proof. Proof of part (1). For the proof that there are no nontrivial solutions to (1.4) if Assumption 1 holds, see [11, Proposition 5 and Remark 1 following it]. Non-explosiveness of $Y(t)$ then follows from the parenthetical sentence following (1.4) above.

Proof of part (2). For the proof that there are no nontrivial solutions to (1.2), see [11, Theorem 2]. The fact that the compact support property holds then follows from Theorem EP1 above.

The conditions in Assumption 1 are classical conditions which arise frequently in the theory of diffusion processes. Theorem EP2 shows that if the coefficients of $L$ obey this condition and if the branching coefficient $\alpha$ is bounded away from zero, then everything is well behaved-neither can the underlying diffusion process explode nor can the measure-valued process fail to possess the compact support property.

The following result shows that the compact support property can fail if $\inf _{x \in \mathbb{R}^{d}} \alpha(x)=0$. It also demonstrates that the effect of $\alpha$ on the compact support property cannot be studied in isolation, but in fact depends on the underlying diffusion.

Theorem 1. Let $p \in(1,2]$ and let

$$
L=\frac{1}{2} \sum_{i, j=1}^{d} a_{i, j} \frac{\partial^{2}}{\partial x_{i} \partial x_{j}}
$$

where

$$
C_{0}^{-1}(1+|x|)^{m} \leqslant \sum_{i, j=1}^{d} a_{i, j}(x) \leqslant C_{0}(1+|x|)^{m}, \quad m \in[0,2], \text { for some } C_{0}>0 .
$$

(1) If

$$
\alpha(x) \geqslant C_{1} \exp \left(-C_{2}|x|^{2-m}\right),
$$

for some $C_{1}, C_{2}>0$, then the compact support property holds for $X(t)$.

(2) If

$$
\alpha(x) \leqslant C \exp \left(-|x|^{2-m+\epsilon}\right) \text { and } \beta(x) \geqslant-C(1+|x|)^{2-m+2 \delta},
$$

for some $C, \epsilon>0$ and some $\delta<\epsilon$, then the compact support property does not hold for $X(t)$.

Remark. By Theorem EP1, to prove Theorem 1, it is necessary and sufficient to show that if $\alpha$ is as in part (1) of the theorem, then there is no nontrivial solution to (1.2) with initial data $f=0$, while if $\alpha$ is as in part (2) of the theorem, then there is such a nontrivial solution. In the case that $L=\frac{1}{2} \Delta$, and for part (2), $\beta \geqslant 0$, this result was obtained in [11, Theorem 7]. An alternative, more purely probabilistic proof which does not rely on Theorem EP1 can be found in [16] for the case $L=\frac{1}{2} \Delta$ and $\beta=0$.

A heuristic, qualitative understanding of Theorem 1 is given at the end of this section.

As a complement to Theorem 1, we note the following result $[9,11]$. 
Theorem EP3. Let $p \in(1,2]$.

(1) Let $d \geqslant 2$ and let $L=A(x) \Delta$, where $A(x) \geqslant C(1+|x|)^{m}$, for some $C>0$ and $m>2$.

Assume that

$$
\sup _{x \in \mathbb{R}^{d}} \alpha(x)<\infty \text { and } \beta \geqslant 0 .
$$

Then the compact support property does not hold for $X(t)$.

(2) Let $d=1$ and let $L=A(x) \frac{\mathrm{d}^{2}}{\mathrm{~d} x^{2}}$, where $A(x) \geqslant C(1+|x|)^{m}$, for some $C>0$ and $m>1+p$.

Assume that

$$
\sup _{x \in \mathbb{R}^{d}} \alpha(x)<\infty \text { and } \beta \geqslant 0 .
$$

Then the compact support property does not hold for $X(t)$.

(3) Let $d=1$ and let $L=A(x) \frac{\mathrm{d}^{2}}{\mathrm{~d} x^{2}}$, where $A(x) \leqslant C(1+|x|)^{m}$, for some $C>0$, and $m \leqslant 1+p$.

Assume that

$$
\inf _{x \in \mathbb{R}^{d}} \alpha(x)>0 \text { and } \beta \leqslant 0 .
$$

Then the compact support property holds for $X(t)$.

Remark 1. It follows from Theorem EP3 that if $d=1$ and $L=(1+|x|)^{m} \frac{\mathrm{d}^{2}}{\mathrm{~d} x^{2}}$, with $m \in(2,3]$, and say $\alpha=1$ and $\beta=0$, then the compact support property will depend on the particular choice of $p \in(1,2]$.

Remark 2. If $d=2$ and $L=(1+|x|)^{m} \Delta$, with $m>2$, and say $\alpha=1$ and $\beta=0$, then by Theorem EP3, the compact support property does not hold, yet the underlying diffusion does not explode since it is a time-change of a recurrent process; namely, of two-dimensional Brownian motion.

Proof. By Theorem EP1, it is necessary and sufficient to show that under the conditions of parts (1) and (2), there exists a nontrivial solution to (1.2), while under the conditions of part (3) there does not. In the case that $\alpha=1$ (or equivalently, any positive constant) and $\beta=0$, this follows from [11, Theorem 5]. To extend this to $\alpha$ and $\beta$ as in the statement of the theorem, one appeals to a comparison result which we state below [11, Proposition 4].

Comparison result. Assume that

$$
\beta_{1} \leqslant \beta_{2}
$$

and

$$
0<\alpha_{2} \leqslant \alpha_{1} .
$$

If uniqueness holds for (1.2) with initial data $f=0$ when $\beta=\beta_{2}$ and $\alpha=\alpha_{2}$, then uniqueness also holds when $\beta=\beta_{1}$ and $\alpha=\alpha_{1}$. Thus, from Theorem EP1, if the compact support property holds for $\beta=\beta_{2}$ and $\alpha=\alpha_{2}$, then it also holds for $\beta=\beta_{1}$ and $\alpha=\alpha_{1}$.

Theorem 1 and Theorem EP3 demonstrate the effect of the underlying diffusion $Y(t)$ on the compact support property in the case that $L$ is comparable to $(1+|x|)^{m} \Delta$. We now consider more generally the effect of the underlying diffusion process on the compact support property. We begin with the following result which combines [11, Theorem 3] with Theorem EP1.

Theorem EP4. Let $p \in(1,2]$ and assume that the underlying diffusion process $Y(t)$ explodes. Assume in addition that

$$
\inf _{x \in \mathbb{R}^{d}} \frac{\beta(x)}{\alpha(x)}>0 .
$$

Then there exists a nontrivial solution to (1.2) with initial data $f=0$; thus, by Theorem EP1, the compact support property does not hold. 
In particular, it follows from Theorem EP4 that if $Y(t)$ is explosive and $\sup _{x \in \mathbb{R}^{d}} \alpha(x)<\infty$, then a sufficient condition for the compact support property to fail is that $\inf _{x \in \mathbb{R}^{d}} \beta(x)>0$. It turns out that this result can be extended significantly. We will prove the following key result.

Theorem 2. Let $p \in(1,2]$ and let $L$ and $\alpha$ be arbitrary. Assume that

$$
\sup _{x \in \mathbb{R}^{d}}\left|\beta_{1}-\beta_{2}\right|<\infty
$$

Then uniqueness holds for (1.2) with initial data $f=0$ for $L, \alpha$ and $\beta_{1}$ if and only if it holds for $L, \alpha$ and $\beta_{2}$. Thus, from Theorem EP1, the compact support property holds with $\beta_{1}$ if and only if it holds with $\beta_{2}$.

Remark. Theorem 2 states that a bounded change in the parameter $\beta$ cannot influence the compact support property.

As an immediate consequence of Theorem EP4 and Theorem 2, we obtain the following result.

Theorem 3. Let $p \in(1,2]$ and assume that the underlying diffusion process $Y(t)$ explodes. Assume that

$$
\sup _{x \in \mathbb{R}^{d}} \alpha(x)<\infty \text { and } \inf _{x \in \mathbb{R}^{d}} \beta(x)>-\infty .
$$

Then there exists a nontrivial solution to (1.2) with initial data $f=0$; thus by Theorem EP1, the compact support property does not hold.

Remark 1. Theorem 3 shows that if the branching mechanism satisfies (1.5), then the compact support property never holds if the underlying diffusion is explosive. The converse is not true-an example was given in Remark 2 following Theorem EP3, and another one appears in Remark 2 following Corollary 1.

Remark 2. Starting from Theorem EP4, a direct probabilistic proof of Theorem 3 can be given in the case $p=2$ via Dawson's Girsanov theorem for super-diffusion processes [3].

Remark 3. Theorem 3 is significant also from the point of view of pde's. It states that under the condition (1.5), nonuniqueness in the class of bounded solutions to the linear equation (1.4) guarantees nonuniqueness for the semilinear equation (1.2) with initial data $f=0$.

The next result shows that the restriction $\sup _{x \in \mathbb{R}^{d}} \alpha(x)<\infty$ in Theorem 3 is essential.

Proposition 1. Let $p \in(1,2]$. Let $m \in(-\infty, \infty)$,

$$
\begin{aligned}
& \quad L=(1+|x|)^{m} \Delta \quad \text { in } \mathbb{R}^{d}, \\
& \beta=0 \text { and } \\
& \alpha(x) \geqslant c(1+|x|)^{m-2},
\end{aligned}
$$

for some $c>0$. Then the compact support property holds for the measure-valued process $X(t)$. However, if $m>2$ and $d \geqslant 3$, the diffusion process $Y(t)$ explodes.

Proof. By the comparison result above, it suffices to consider the case that $\alpha=c(1+|x|)^{m-2}$. Note that upon dividing by $(1+|x|)^{m}$, the stationary elliptic equation can be written in the form $\Delta W-\frac{c}{(1+|x|)^{2}} W^{p}=0$ in $\mathbb{R}^{d}$. There is no nontrivial, nonnegative solution to this equation (see, for example [11, Theorem 6]). From this it follows that uniqueness holds for (1.2) with initial data $f=0$; indeed, if uniqueness did not hold, and $u(x, t)$ were a nontrivial solution, then it would follow from the maximum principle that $u(x, t)$ is increasing in $t$. Then $W(x) \equiv \lim _{t \rightarrow \infty} u(x, t)$ would constitute a nontrivial solution to the above stationary elliptic equation. (For details, see [11, Theorem 4(ii)].) Since uniqueness holds for (1.2), it follows from Theorem EP1 that the compact support property holds. For a proof that $Y(t)$ explodes if $m>2$ and $d \geqslant 3$, see, for example, [11, Proposition 5 and Remark 1 following it]. 
As mentioned in the introduction, $p$ and $b$ play only a minor role in determining whether or not the compact support property holds. Theorem 2 demonstrates the limited role played by $\beta$. In Remark 1 following Theorem EP3, we have seen a rather restricted example where $p$ can effect the compact support property. In the sequel we will give another example where $p$ can effect the compact support property and also an example where $\beta$ can effect the compact support property. In order to accomplish this, we first need to discuss how the concept of a measure-valued process hitting a point can be formulated and understood in terms of the compact support property. This last point is of independent interest.

Let $\mathcal{R}_{t}=\operatorname{cl}\left(\bigcup_{s \in[0, t]} \operatorname{supp}(X(s))\right)$ and let $\mathcal{R}=\operatorname{cl}\left(\bigcup_{s \geqslant 0} \operatorname{supp}(X(s))\right)$. The random set $\mathcal{R}$ is called the range of $X=X(\cdot)$. A path of the measure-valued process is said to hit a point $x_{0} \in \mathbb{R}^{d}$ if $x_{0} \in \mathcal{R}$. If $X(t)$ becomes extinct with probability one, that is, $P_{\mu}(X(t)=0$ for all large $t)=1$, or more generally, if $X(t)$ becomes locally extinct with probability one, that is, $P_{\mu}(X(t, B)=0$ for all large $t)=1$, for each bounded $B \subset \mathbb{R}^{d}$, then $x_{0} \in \mathcal{R}$ if and only if $x_{0} \in \mathcal{R}_{t}$ for sufficiently large $t$. Thus, we have:

If $X(t)$ suffers local extinction with probability one, and if the compact support property holds for $X(t)$ on $\mathbb{R}^{d}$, then $P_{\mu}\left(X\right.$ hits $\left.x_{0}\right)>0$ if and only if there exists a $t>0$ such that

$P_{\mu}\left(\bigcup_{0 \leqslant s \leqslant t} \operatorname{supp}(X(s))\right.$ is not compactly embedded in $\left.\mathbb{R}^{d}-\left\{x_{0}\right\}\right)>0$.

Now although we have assumed in this paper that the underlying state space is $\mathbb{R}^{d}$, everything goes through just as well on an arbitrary domain $D \subset \mathbb{R}^{d}[10]$. Of course now, the compact support property is defined with respect to the domain $D$, and the underlying diffusion will explode if it hits $\partial D$ or runs out to $\infty$ in finite time. In particular, Theorem EP1 still holds with $\mathbb{R}^{d}$ replaced by $D[10]$.

In light of the above observations, consider a measure-valued process $X(t)$ corresponding to the log-Laplace equation (1.2) on $\mathbb{R}^{d}$ with $d \geqslant 2$. The underlying diffusion process $Y(t)$ on $\mathbb{R}^{d}$ corresponds to the operator $L$ on $\mathbb{R}^{d}$. Let $\widehat{Y}(t)$ denote the diffusion process on the domain $D=\mathbb{R}^{d}-\left\{x_{0}\right\}$ with absorption at $x_{0}$ and corresponding to the same operator $L$. (Note that if $x_{0}$ is polar for $Y(t)$, then $Y(t)$ and $\widehat{Y}(t)$ coincide when started from $x \neq x_{0}$. In fact, $x_{0}$ is always polar under the assumptions we have placed on the coefficients of $L$ [5].) Let $\widehat{X}(t)$ denote the measure-valued process corresponding to the log-Laplace equation (1.2), but with $\mathbb{R}^{d}$ replaced by $D=\mathbb{R}^{d}-\left\{x_{0}\right\}$. It follows from (1.6) that if $X(t)$ suffers local extinction with probability one, and if the compact support property holds for $X(t)$ on $\mathbb{R}^{d}$, then the measure-valued process $X(t)$ hits the point $x_{0}$ with positive probability if and only if $\widehat{X}(t)$ on $\mathbb{R}^{d}-\left\{x_{0}\right\}$ does not possess the compact support property. Furthermore, the above discussion shows that even if $X(t)$ does not suffer local extinction with probability one, a sufficient condition for $X(t)$ to hit the point $x_{0}$ with positive probability is that $\widehat{X}(t)$ on $\mathbb{R}^{d}-\left\{x_{0}\right\}$ does not possess the compact support property. Similarly, even if the compact support property does not hold for $X(t)$ on $\mathbb{R}^{d}$, a necessary condition for $X(t)$ to hit the point $x_{0}$ with positive probability is that $\widehat{X}(t)$ on $\mathbb{R}^{d}-\left\{x_{0}\right\}$ does not possess the compact support property.

A similar analysis can be made when $d=1$. The process $\widehat{X}(t)$ above must be replaced by two processes, $\widehat{X}^{+}(t)$ and $\widehat{X}^{-}(t)$, defined respectively on $\left(x_{0}, \infty\right)$ and $\left(-\infty, x_{0}\right)$. The claim in italics above then holds with the requirement on $\widehat{X}(t)$ transferred to both $\widehat{X}^{+}(t)$ and $\widehat{X}^{-}(t)$. In the sequel, we will assume that $d \geqslant 2$.

Consider now the following semi-linear equation in the punctured space $\mathbb{R}^{d}-\{0\}$.

$$
\begin{aligned}
& u_{t}=\frac{1}{2} \Delta u-u^{p} \quad \text { in }\left(\mathbb{R}^{d}-\{0\}\right) \times(0, \infty) ; \\
& u(x, 0)=0 \quad \text { in } \mathbb{R}^{d}-\{0\} \\
& u \geqslant 0 \quad \text { in }\left(\mathbb{R}^{d}-\{0\}\right) \times[0, \infty) .
\end{aligned}
$$

By Theorem EP1, the measure-valued process corresponding to the semi-linear equation

$$
u_{t}=\frac{1}{2} \Delta u-u^{p} \quad \text { in } \mathbb{R}^{d}-\{0\}
$$

will possess the compact support property if and only if (1.7) has a nontrivial solution. The following theorem was recently proved directly in [15]; in fact it is a particular case of a more general result in [1]. 
Theorem BP. Let $p>1$ and $d \geqslant 2$.

1. If $d<\frac{2 p}{p-1}$, then there exists a nontrivial solution to (1.7).

2. If $d \geqslant \frac{2 p}{p-1}$, then there is no nontrivial solution to (1.7).

Remark. In Theorem BP, $p>1$ is unrestricted, even though of course there is no probabilistic import when $p>2$.

The following result will be proved by the method used in [15] to prove Theorem BP.

Theorem 4. Let $p>1$ and $d \geqslant 2$. Let $X(t)$ denote the measure-valued process on the punctured space $\mathbb{R}^{d}-\{0\}$ corresponding to the semi-linear equation $u_{t}=\frac{1}{2} \Delta u+\beta u-u^{p}$ in $\left(\mathbb{R}^{d}-\{0\}\right) \times(0, \infty)$. Consider the Cauchy problem

$$
\begin{aligned}
& u_{t}=\frac{1}{2} \Delta u+\beta u-u^{p} \quad \text { in }\left(\mathbb{R}^{d}-\{0\}\right) \times(0, \infty) ; \\
& u(x, 0)=0 \quad \text { in } \mathbb{R}^{d}-\{0\} ; \\
& u \geqslant 0 \quad \text { in }\left(\mathbb{R}^{d}-\{0\}\right) \times[0, \infty) .
\end{aligned}
$$

Assume that

$$
d<\frac{2 p}{p-1} .
$$

Let

$$
\beta_{0}=\frac{d(p-1)-2 p}{(p-1)^{2}}<0 .
$$

(1) If

$$
\beta(x) \geqslant \frac{\beta_{0}+\kappa}{|x|^{2}}, \quad \text { for some } \kappa \in\left(0,-\beta_{0}\right],
$$

then there exists a nontrivial solution to (1.8); hence, the compact support property does not hold for $X(t)$.

(2) If

$$
\limsup _{x \rightarrow 0}|x|^{2} \beta(x)<\beta_{0}
$$

then there is no nontrivial solution to (1.8); hence, the compact support property holds for $X(t)$.

Remark. The restriction $\kappa \leqslant-\beta_{0}$ is made to ensure that $\beta$ is bounded from above.

We have the following corollary of Theorem BP and Theorem 4.

Corollary 1. Let $X(t)$ denote the measure-valued process on all of $\mathbb{R}^{d}, d \geqslant 2$, corresponding to the semi-linear equation

$$
u_{t}=\frac{1}{2} \Delta u+\beta u-u^{p} \quad \text { in } \mathbb{R}^{d} \times(0, \infty) .
$$

(1) If $\beta$ is bounded from below and $d<\frac{2 p}{p-1}$, then $X(t)$ hits a fixed point $x_{0}$ with positive probability;

(2) If $\beta \leqslant 0$ and $d \geqslant \frac{2 p}{p-1}$, then $X(t)$ hits a fixed point $x_{0}$ with probability 0 ;

(3) If $\beta \leqslant 0, d<\frac{2 p}{p-1}$ and $\beta$ has a singularity at the origin such that

$$
\limsup _{x \rightarrow 0}|x|^{2} \beta(x)<\frac{d(p-1)-2 p}{(p-1)^{2}},
$$

then $X(t)$ hits 0 with probability 0 . 
Proof. When $\beta=0$, part (1) follows immediately from Theorem BP, Theorem EP1, the discussion preceding Theorem BP and the fact that the compact support property holds for $X(t)$ on $\mathbb{R}^{d}$. For the general case one appeals to Theorem 2, which holds just as well for a punctured space. (Recall that we are always assuming in this paper that $\beta$ is bounded.)

When $\beta=0$, it is well known that the super-Brownian motion in the statement of the corollary suffers extinction with probability one. By comparison, this also holds when $\beta \leqslant 0$ [10]. Thus, part (2) follows from Theorem BP, Theorem EP1 and the discussion preceding Theorem BP, while part (3) follows from Theorem 4, Theorem EP1 and the discussion preceding Theorem BP.

Remark 1. When $\beta=0$, the results in parts (1) and (2) of Corollary 1 state that critical, super-Brownian motion hits a point with positive probability if $d<\frac{2 p}{p-1}$, and with zero probability if $d \geqslant \frac{2 p}{p-1}$. This result can be found in [4] for the case $p=2$. For $p \in(1,2]$, it can be found in [6] or in [8], which exploit the method of removable singularities for elliptic equations, developed in [2] and [17]. The approach here is via the parabolic equation, which is more in the line of the approach used in [7] for the study of space-time polar sets.

Remark 2. Theorem BP gives an example where the measure-valued process does not possess the compact support property even though the underlying diffusion process does not explode. Indeed, the underlying diffusion is Brownian motion in $\mathbb{R}^{d}-\{0\}$. Since singletons are polar for multi-dimensional Brownian motion, this process does not explode. However for $d<\frac{2 p}{p-1}$, the compact support property fails for the measure-valued process.

Remark 3. In order to obtain an example of the phenomenon occurring in Remark 2 when the state space is the whole space, and in order to see how the parameter $p$ and the mass creation parameter $\beta$ can affect the compact support property when the state space is the whole space, we convert the set-up in Theorem 4 and Theorem BP to the state space $\mathbb{R}=(-\infty, \infty)$ by considering just the radial variable, $r=|x|$, and then making a change of variables, say, $z=\frac{1}{r}-r$. One obtains an operator $L=\frac{1}{2} a(z) \frac{\mathrm{d}^{2}}{\mathrm{~d} z^{2}}+b(z) \frac{\mathrm{d}}{\mathrm{d} z}$ on $\mathbb{R}$, where

$$
\begin{aligned}
& a(z) \sim z^{4} \text { as } z \rightarrow \infty, \quad \lim _{z \rightarrow-\infty} a(z)=1 ; \\
& b(z) \sim \frac{3-d}{2} z^{3}, \quad \text { as } z \rightarrow \infty, \quad \lim _{z \rightarrow-\infty} b(z)=0 .
\end{aligned}
$$

Also, one has $\alpha=1$. One has $\beta=0$ in Theorem BP and one has

$$
\begin{aligned}
& \beta(z) \geqslant \frac{4\left(\beta_{0}+\kappa\right)}{\left(\left(z^{2}+4\right)^{1 / 2}-z\right)^{2}} \quad \text { in part (1) of Theorem } 4 \\
& \limsup _{z \rightarrow \infty} \frac{1}{4}\left(\left(z^{2}+4\right)^{1 / 2}-z\right)^{2} \beta(z)<\beta_{0} \quad \text { in part (2) of Theorem } 4 .
\end{aligned}
$$

It follows then that $\liminf _{z \rightarrow \infty} \beta(z) / z^{2} \geqslant \beta_{0}+\kappa$ in part (1) of Theorem 4 and $\lim \sup _{z \rightarrow \infty} \beta(z) / z^{2}<\beta_{0}$ in part (2) of Theorem 4.

Consider first the phenomenon mentioned in Remark 2 with regard to Theorem BP. After the change of variables, the state space is $\mathbb{R}$ and the operator $L$ on $\mathbb{R}$ depends on the parameter $d$. The one-dimensional diffusion corresponding to $L$ is nonexplosive for all $d \geqslant 2$, as it inherits this property from the original process before the change of variables. Also $\alpha=1$ and $\beta=0$. However, if $d<\frac{2 p}{p-1}$, then the compact support property fails. (Another example of this phenomenon was presented in Remark 2 following Theorem EP3.)

With the same setup as in the previous paragraph, we also see how the parameter $p$ affects the compact support property-the property will hold if and only if $p \geqslant \frac{d}{d-2}$.

Now consider the above change of variables applied to Theorem 4. We see that if $d<\frac{2 p}{p-1}$, then $\beta$ affects the compact support property-it holds if $\beta$ satisfies (1.9b) and does not hold if $\beta$ satisfies (1.9a). As Theorem 2 guarantees, an unbounded change in $\beta$ was needed to effect a change in the compact support property.

Theorems 1, 2 and 4 will be proved successively in the sections that follow.

We now turn to an intuitive probabilistic understanding of the role of the branching in Theorem 1 . We recall the particle process approximation to the measure-valued process in the case that $\alpha$ and $\beta$ are bounded. Consider first the 
case $p=2$, the case in which the offspring distribution has finite variance. Let $\widehat{\mathbb{R}}^{d}=\mathbb{R}^{d} \cup\{\Delta\}$ denote the one-point compactification of $\mathbb{R}^{d}$. One may consider the diffusion process $Y(t)$ to live on $\widehat{\mathbb{R}}^{d}$; if $Y(t)$ does not explode, then it never reaches $\Delta$, while if $Y(t)$ does explode, then it enters the state $\Delta$ upon leaving $\mathbb{R}^{d}$, and remains there forever. For each positive integer $n$, consider $N_{n}$ particles, each of mass $\frac{1}{n}$, starting at points $y_{i}^{(n)}(0) \in \mathbb{R}^{d}, i=1,2, \ldots, N_{n}$, and performing independent branching diffusion according to the process $Y(t)$, with branching rate $c n, c>0$, and spatially dependent branching distribution $\left\{p_{k}^{(n)}(y)\right\}_{k=0}^{\infty}$, where

$$
\begin{aligned}
& \sum_{k=0}^{\infty} k p_{k}^{(n)}(y)=1+\frac{\gamma(y)}{n}+\mathrm{o}\left(\frac{1}{n}\right), \quad \text { as } n \rightarrow \infty ; \\
& \sum_{k=0}^{\infty}(k-1)^{2} p_{k}^{(n)}(y)=m(y)+o(1), \quad \text { as } n \rightarrow \infty, \text { uniformly in } y,
\end{aligned}
$$

with $m, \gamma \in C^{\alpha}\left(\mathbb{R}^{d}\right)$ and $m(y)>0$. Let $N_{n}(t)$ denote the number of particles alive at time $t$ and denote their positions by $\left\{Y_{i}^{(n)}(t)\right\}_{i=1}^{N_{n}(t)}$. Denote by $\mathcal{M}_{F}\left(\mathbb{R}^{d}\right)\left(\mathcal{M}_{F}\left(\widehat{\mathbb{R}}^{d}\right)\right)$ the space of finite measures on $\mathbb{R}^{d}\left(\widehat{\mathbb{R}}^{d}\right)$. Define an $\mathcal{M}_{F}\left(\widehat{\mathbb{R}}^{d}\right)$ valued process $X_{n}(t)$ by $X_{n}(t)=\frac{1}{n} \sum_{1}^{N_{n}(t)} \delta_{Y_{i}^{(n)}(t)}(\cdot)$. Note that $X_{n}$ is càdlàg. Denote by $P^{(n)}$ the probability measure corresponding to $\left\{X_{n}(t), 0 \leqslant t<\infty\right\}$ on $D\left([0, \infty), \mathcal{M}_{F}\left(\widehat{\mathbb{R}}^{d}\right)\right)$, the space of càdlàg paths with the Skorohod topology.

Assume that $m(y)$ and $\gamma(y)$ are bounded from above. One can show that if $w-\lim _{n \rightarrow \infty} X_{n}(0)=\mu \in$ $\mathcal{M}_{F}\left(\mathbb{R}^{d}\right)$, then $P_{\mu}^{*}=w-\lim _{n \rightarrow \infty} P^{(n)}$ exists in $D\left([0, \infty), \mathcal{M}_{F}\left(\widehat{\mathbb{R}}^{d}\right)\right)$. Furthermore, the measure $P_{\mu}^{*}$ restricted to $D\left([0, \infty), \mathcal{M}_{F}\left(\mathbb{R}^{d}\right)\right)$ satisfies (1.1) and (1.2) with $\beta(y)=c \gamma(y), \alpha(y)=\frac{1}{2} c m(y)$ and $p=2$ (see [10]). Denoting this restriction by $\left.P_{\mu}^{*}\right|_{\mathbb{R}^{d}}$, it then follows that $P_{\mu}=\left.P_{\mu}^{*}\right|_{\mathbb{R}^{d}}$. In fact, one can show that $P_{\mu}$ is supported on the space of continuous paths, $C\left([0, \infty), \mathcal{M}_{F}\left(\mathbb{R}^{d}\right)\right)$.

One should think of $\beta$ and $\alpha$ as the mass creation and the variance parameters respectively of the branching.

For the case that $p \in(1,2)$, one cooks up a sequence of distributions $\left\{p_{k}^{(n)}(y)\right\}_{n=1}^{\infty}$ for which the generating functions $\Phi^{(n)}(s ; y)=\sum_{k=0}^{\infty} p_{k}^{(n)}(y) s^{y}$ satisfy $\lim _{n \rightarrow \infty} n^{p}\left(\Phi^{(n)}\left(1-\frac{\lambda}{n} ; y\right)-\left(1-\frac{\lambda}{n}\right)\right)=\alpha(y) \lambda^{p}-\beta(y) \lambda$. These offspring distributions $\left\{p_{k}^{(n)}(y)\right\}_{n=1}^{\infty}$ will possess all moments smaller than $p$. Again, $\beta$ can be thought of as the mass creation parameter. As above, each particle is given mass $\frac{1}{n}$, but in the present case, the branching rate is $n^{p-1}$. The same construction and conclusion as above holds, although in this case the paths are not continuous, but only càdlàg [7].

With the above set-up, we can now give some intuition concerning Theorem 1. Consider two particular cases of the above construction. In both cases we will assume that at time 0 there are $n$ particles, all positioned at $y=0$; that is, $N_{n}=n$ and $y_{i}^{(n)}=0$, for $i=1,2, \ldots n$. Then the initial measure, both for the approximating process and the limiting one, will be $\mu=\delta_{0}$. We will also assume that the diffusion $Y(t)$ does not explode.

The first case is the completely trivial case in which there is no branching at all. This degenerate case corresponds to $\beta=\alpha=0$ (and thus does not actually fit into the above set-up). In this case, $X_{n}(t)$ is a random probability measure with $n$ atoms of mass $\frac{1}{n}$ positioned at $n$ IID points, distributed according to the distribution of $Y(t)$. Thus, by the law of large numbers, $X(t)=w-\lim _{n \rightarrow \infty} X_{n}(t)$ is the deterministic measure $\operatorname{dist}(Y(t))$. Since $\operatorname{dist}(Y(t))$ is not compactly supported for $t>0$, it follows that the compact support property does not hold for $X(t)$ in this trivial case.

Now consider the case of critical, binary branching; that is, $p_{0}^{(n)}=p_{2}^{(n)}=\frac{1}{2}$. Letting $c=1$, it then follows that $\beta=0$ and $\alpha=\frac{1}{2}$. In this case, it is well known that for any $s<1$, if one lets $p_{n}(s, M)$ denote the probability that all the mass at time 1 in the approximate measure-valued process $X_{n}(\cdot)$ descends from no more than $M$ ancestors alive at time $s$, then $\lim _{M \rightarrow \infty} \lim _{n \rightarrow \infty} p_{n}(s, M)=1$. Thus, since all the particles alive at time 1 are coming from a finite number of ancestors at any time $s$, these particles are correlated, and the law of large numbers does not apply, allowing for compact support property to hold.

The above discussion suggests that one way for the compact support property to break down is for the branching mechanism to be spatially dependent and to decay sufficiently fast as $|x| \rightarrow \infty$ so that the law of large numbers will come into play. Furthermore, the faster the diffusion is, the more quickly individual particles that begin together become statistically uncorrelated, so one might expect that the stronger the diffusion, the weaker the threshold on the decay rate in order for the compact support property to break down. If the diffusion process $Y(t)$ corresponds to the operator $L=\frac{1}{2}(1+|x|)^{m} \Delta$, for $m \in[0,2]$, then for $m=0$ one obtains Brownian motion, while for $m \in(0,2]$, one obtains a time-changed Brownian motion with the diffusion sped up, the speed increasing in $m$. Theorem 1 shows 
that for such an underlying diffusion, for any $\epsilon>0$, the rate $\exp \left(-|x|^{2-m+\epsilon}\right)$ is sufficiently fast to cause the compact support property to fail, but the rate $\exp \left(-|x|^{2-m}\right)$ is not fast enough.

\section{Proof of Theorem 1}

To make the calculations simpler, we will prove the theorem in the case that $L=A(x) \Delta$, where $C_{0}^{-1}(1+|x|)^{m} \leqslant$ $A(x) \leqslant C_{0}(1+|x|)^{m}$, for some $m \in[0,2]$. The general case follows in the same fashion.

Proof of part (1). Let $u(x, t)$ be any solution of (1.2) with initial data $g=0$. By Theorem EP1, we need to show that $u \equiv 0$. Define $\widehat{U}(x, t)$ through the equality $u(x, t)=\widehat{U}(x, t) \exp \left(\lambda\left(1+|x|^{2}\right)^{(2-m) / 2}(t+\delta)\right)$, for some $\lambda, \delta>0$. Then

$$
\begin{aligned}
\exp ( & \left.-\lambda\left(1+|x|^{2}\right)^{(2-m) / 2}(t+\delta)\right) A(x) \Delta u \\
= & A(x)\left(\Delta \widehat{U}+2 \lambda(2-m)\left(1+|x|^{2}\right)^{-m / 2}(t+\delta) x \cdot \nabla \widehat{U}\right)+A(x)\left((t+\delta)^{2} \lambda^{2}(2-m)^{2}\left(1+|x|^{2}\right)^{-m}|x|^{2}\right. \\
& \left.+(t+\delta) \lambda d(2-m)\left(1+|x|^{2}\right)^{-m / 2}-(t+\delta) \lambda(2-m) m\left(1+|x|^{2}\right)^{-m / 2-1}|x|^{2}\right) \widehat{U} .
\end{aligned}
$$

Also, using the bound on $\alpha$ in the statement of the theorem, we have

$$
\begin{aligned}
& \exp \left(-\lambda\left(1+|x|^{2}\right)^{(2-m) / 2}(t+\delta)\right)\left(\beta u-\alpha u^{p}-u_{t}\right) \\
& \quad=\left(\beta-\lambda\left(1+|x|^{2}\right)^{(2-m) / 2}\right) \widehat{U}-\alpha \exp \left((p-1) \lambda\left(1+|x|^{2}\right)^{(2-m) / 2}(t+\delta)\right) \widehat{U}^{p} .
\end{aligned}
$$

Since $u$ is a solution to (1.2), the sum of the left-hand sides of (2.1) and (2.2) is equal to 0 . Consider now the sum of the terms on the right-hand sides of (2.1) and (2.2) with the variable $t$ restricted by $0 \leqslant t \leqslant \delta$. By assumption, $\alpha(x) \geqslant C_{1} \exp \left(-C_{2}|x|^{2-m}\right)$. Thus, the coefficient of $\widehat{U}^{p}$ will be bounded away from 0 if

$$
\lambda \delta=\frac{C_{2}}{p-1} .
$$

In the case that $m=2$, the coefficient of $\widehat{U}$ is bounded from above. Otherwise, the two unbounded terms in the coefficient of $\widehat{U}$ are $A(x)(t+\delta)^{2} \lambda^{2}(2-m)^{2}\left(1+|x|^{2}\right)^{-m}|x|^{2}$ and $-\lambda\left(1+|x|^{2}\right)^{(2-m) / 2}$. By assumption, there exists a $C_{4}>0$ such that $A(x) \leqslant C_{4}\left(1+|x|^{2}\right)^{m / 2}$. Thus, in order to guarantee that the coefficient of $\widehat{U}$ is bounded from above, it suffices to have $\lambda=C_{4}(2 \delta)^{2} \lambda^{2}(2-m)^{2}$. Using (2.3) to substitute for $(\lambda \delta)^{2}$ on the right-hand side above, we have

$$
\lambda=4 C_{4}(2-m)^{2} C_{2}^{2}(p-1)^{-2} .
$$

With $\lambda$ and $\delta$ chosen as in (2.3) and (2.4), it then follows

$$
\begin{aligned}
& A(x)\left(\Delta \widehat{U}+2 \lambda(2-m)\left(1+|x|^{2}\right)^{-m / 2}(t+\delta) x \cdot \nabla \widehat{U}\right)+c_{1} \widehat{U}-c_{2} \widehat{U}^{p} \geqslant 0, \\
& \quad \text { for }(x, t) \in \mathbb{R}^{n} \times[0, \delta], \text { for some } c_{1}, c_{2}>0 .
\end{aligned}
$$

Let $M_{R, K}(x, t)=(1+|x|)^{2 /(p-1)}(R-|x|)^{-2 /(p-1)} \exp (K(t+1))$, for $(x, t) \in B_{R} \times(0, \infty)$. In [11, Proof of Theorem 2], it was shown that for any operator $\mathcal{L}$ satisfying the conditions of Theorem EP2, there exists a $K>0$ such that for all $R>0$

$$
\mathcal{L} M_{R, K}(x, t) \leqslant 0, \quad(x, t) \in B_{R} \times(0, \infty),
$$

where $B_{R}$ denotes the ball of radius $R$ centered at the origin. In particular the operator

$$
\mathcal{A}=A(x)\left(\Delta+2 \lambda(2-m)\left(1+|x|^{2}\right)^{-m / 2}(t+\delta) x \cdot \nabla\right)
$$

satisfies the conditions of Theorem EP2, except for the fact that it is time inhomogeneous. The time inhomogeneity causes no problem since we are only considering $t \in[0, \delta]$ and since for fixed $x$, everything in sight is uniformly bounded for $t \in[0, \delta]$. Thus the proof of (2.6) shows that

$$
\mathcal{A} M_{R, K}(x, t) \leqslant 0, \quad(x, t) \in B_{R} \times(0, \delta] .
$$

Since $0=\widehat{U}(x, 0) \leqslant M_{R, K}(x, 0)$ and $\widehat{U}(y, t) \leqslant M_{R, K}(y, t)=\infty$, for $y \in \partial B_{R}$, it follows from (2.5), (2.7) and the maximum principle for semi-linear equations [11, Proposition 1] that

$$
\widehat{U}(x, t) \leqslant M_{R, K}(x, t), \quad \text { for }(x, t) \in B_{R} \times[0, \delta] .
$$


Letting $R \rightarrow \infty$, we conclude that $\widehat{U}(x, t)=0$, for $(x, t) \in \mathbb{R}^{d} \times(0, \delta]$. Thus, we also have $u(x, t)=0$, for $(x, t) \in$ $\mathbb{R}^{d} \times[0, \delta]$. Since $u$ satisfies a time homogeneous equation, we conclude that in fact $u(x, t)=0$, for $(x, t) \in \mathbb{R}^{d} \times$ $[0, \infty)$. This completes the proof of part (1).

Proof of part (2). By Theorem EP1, we need to show that there is a nontrivial solution to (1.2) with initial data $g=0$. Let $u(x, t)$ be any solution of (1.2) with initial data $g=0$. Define $\widehat{U}(x, t)$ through the equality $u(x, t)=$ $\widehat{U}(x, t) \exp \left(\lambda\left(1+|x|^{2}\right)^{(2-m+\kappa) / 2}\right)$, where $\kappa \in(\delta, \epsilon)$, and $\epsilon$ and $\delta$ are as in the statement of the theorem. Now (2.1) and (2.2) hold with the following changes: (i) $m$ is replaced by $m-\kappa$; (ii) $(t+\delta)$ is replaced by 1; (iii) the term $\lambda\left(1+|x|^{2}\right)^{(2-m) / 2}$ in (2.2) is deleted. As before, the sum of the left-hand sides of (2.1) and (2.2) is equal to 0 . By assumption, $\alpha(x) \leqslant C \exp \left(-|x|^{2-m+\epsilon}\right)$. Thus, the coefficient of $\widehat{U}^{p}$ in the amended version of (2.2) is bounded from above, since $\kappa<\epsilon$. The coefficient of $\widehat{U}$ in the amended version of (2.2) is $\beta$, and by assumption, there exists a $C_{5}>0$ such that $\beta \geqslant-C_{5}\left(1+|x|^{2}\right)^{(2-m+2 \delta) / 2}$. The coefficient of $\widehat{U}$ in the amended version of (2.1) is

$$
\begin{aligned}
& A(x)\left(\lambda^{2}(2-m+\kappa)^{2}\left(1+|x|^{2}\right)^{-m+\kappa}|x|^{2}+\lambda d(2-m+\kappa)\left(1+|x|^{2}\right)^{-(m-\kappa) / 2}\right. \\
& \left.\quad-\lambda(2-m+\kappa)(m-\kappa)\left(1+|x|^{2}\right)^{-(m-\kappa) / 2-1}|x|^{2}\right) .
\end{aligned}
$$

By assumption, there exists a $C_{3}>0$ such that $A(x) \geqslant C_{3}\left(1+|x|^{2}\right)^{m / 2}$. It is easy to check that by choosing $\lambda$ sufficiently large, the factor multiplying $A(x)$ above will be bounded from below by $\left(2 C_{5} / C_{3}\right)\left(1+|x|^{2}\right)^{(2-2 m+2 \kappa) / 2}$. (For $|x| \leqslant \frac{1}{2}$, use the second term in the parentheses, and for $|x|>\frac{1}{2}$ use the first and third terms.) Thus, the coefficient of $\widehat{U}$ in the amended version of (2.1) is greater or equal to $2 C_{5}\left(1+|x|^{2}\right)^{(2-m+2 \kappa) / 2}$. Since $\kappa>\delta$, it follows that the coefficient of $\widehat{U}$ from the sum of the right-hand sides of (2.1) and (2.2) is bounded below by a positive constant.

The above analysis shows that $\widehat{U}$ satisfies the equation

$$
\begin{aligned}
& \widehat{U}_{t}=A(x)\left(\Delta \widehat{U}+2 \lambda(2-m+\kappa)\left(1+|x|^{2}\right)^{-(m-\kappa) / 2} x \cdot \nabla \widehat{U}\right)+\widehat{\beta} \widehat{U}-\hat{\alpha} \widehat{U}^{p} \\
& \widehat{U}(x, 0)=0
\end{aligned}
$$

where $\hat{\beta} \geqslant C_{6}>0$ and $\hat{\alpha} \leqslant C_{7}$, and that uniqueness for the original equation is equivalent to uniqueness for (2.9). We will show below that the diffusion process corresponding to the operator

$$
A(x)\left(\Delta+2 \lambda(2-m+\kappa)\left(1+|x|^{2}\right)^{-(m-\kappa) / 2} x \cdot \nabla\right)
$$

explodes. Thus, it follows from Theorem EP4 that uniqueness does not hold for (2.9). Consequently, uniqueness does not hold for the original equation with initial data $g=0$; thus, by Theorem EP1, the compact support property does not hold.

It remains to show that the diffusion corresponding to the operator $A(x)\left(\Delta+2 \lambda(2-m+\kappa)\left(1+|x|^{2}\right)^{-(m-\kappa) / 2} x \cdot \nabla\right)$ explodes. The diffusion in question is a time change of the diffusion corresponding to

$$
\Delta+2 \lambda(2-m+\kappa)\left(1+|x|^{2}\right)^{-(m-\kappa) / 2} x \cdot \nabla .
$$

It is not hard to show that since $A(x) \geqslant C_{3}\left(1+|x|^{2}\right)^{m / 2}$, explosion will occur for the diffusion in question if it occurs for the diffusion corresponding to the operator

$$
C_{3}\left(1+|x|^{2}\right)^{m / 2}\left(\Delta+2 \lambda(2-m+\kappa)\left(1+|x|^{2}\right)^{-(m-\kappa) / 2} x \cdot \nabla\right) .
$$

This latter operator is radially symmetric, and its radial component is of the form $p(r) \frac{\mathrm{d}^{2}}{\mathrm{~d} r^{2}}+q(r) \frac{\mathrm{d}}{\mathrm{d} r}$, with $p(r)$ satisfying $c_{1}(1+r)^{m} \leqslant p(r) \leqslant c_{2}(1+r)^{m}$, for all $r \geqslant 0$, and $q(r)$ satisfying $c_{3}(1+r)^{1+\kappa} \leqslant q(r) \leqslant c_{4}(1+r)^{1+\kappa}$, for $r>1$, where $c_{1}, c_{2}, c_{3}, c_{4}>0$. This diffusion explodes by the Feller criterion [14, Theorem 5.1.5].

\section{Proof of Theorem 2}

Proof. By assumption, there exists a $B>0$ such that $\beta_{2} \leqslant \beta_{1}+B$. For $i=1,2$, consider the following parabolic equations:

$$
\begin{aligned}
& u_{t}=L u+\beta_{i} u-\alpha u^{p} \quad \text { on } \mathbb{R}^{d} \times(0, \infty) \\
& u(x, 0)=0, \quad x \in \mathbb{R}^{d} \\
& u \geqslant 0 .
\end{aligned}
$$


To prove the theorem it suffices to show that if (3.1) has a non-zero solution when $\beta_{i}=\beta_{2}$, then it also has a non-zero solution when $\beta_{i}=\beta_{1}$.

Suppose to the contrary that a non-zero solution to (3.1) exists when $\beta_{i}=\beta_{2}$ but not when $\beta_{i}=\beta_{1}$. Let $B_{m}$ denote the ball of radius $m$ centered at the origin in $\mathbb{R}^{d}$. For $i=1,2$, consider the functions $u_{m}^{(i)}, m=1,2, \ldots$ (constructed in [10] for $p=2$; see also [11] for $p \in(1,2])$, where $u_{m}^{(i)}$ solves the equation

$$
\begin{aligned}
& \frac{\partial u_{m}}{\partial t}=L u_{m}+\beta_{i} u-\alpha u_{m}^{p} \quad \text { on } B_{m} \times(0, \infty) ; \\
& \lim _{x \rightarrow \partial B_{m}} u_{m}(x, t)=\infty, \quad t>0 ; \\
& u_{m}(x, 0)=0, \quad x \in \mathbb{R}^{d} \\
& u_{m} \geqslant 0 .
\end{aligned}
$$

Since we have assumed that when $\beta_{i}=\beta_{1}$, the only solution to (3.1) is the zero function, it follows from the construction in $[10,11]$, that

$$
\lim _{m \rightarrow \infty} u_{m}^{(1)}=0 .
$$

The same construction shows that since we have assumed that there exists a non-zero solution to (3.1) when $\beta_{i}=\beta_{2}$, we have

$$
\lim _{m \rightarrow \infty} u_{m}^{(2)} \neq 0 .
$$

Define $v_{m}(x, t)=\mathrm{e}^{B t} u_{m}^{(1)}(x, t)$ on $B_{m} \times(0, \infty)$. Using the fact that $B>0$ and $p>1$ along with (3.2) gives

$$
L v_{m}+\beta_{2} v_{m}-v_{m}^{p}-\frac{\partial v_{m}}{\partial t} \leqslant L v_{m}+\left(\beta_{1}+B\right) v_{m}-v_{m}^{p}-\frac{\partial v_{m}}{\partial t} \leqslant 0 .
$$

We have the boundary and initial conditions

$$
\begin{aligned}
& \lim _{x \rightarrow \partial B_{m}} v_{m}(x, t)=\infty, \quad t>0 ; \\
& v_{m}(x, 0)=0, \quad x \in B_{m} .
\end{aligned}
$$

Then an application of the semi-linear parabolic maximum principle [11, Proposition 1] gives

$$
v_{m} \geqslant u_{m}^{(2)} \text {. }
$$

(In fact, one has compare $v_{m}$ to $u_{n, m}^{(2)}$, where $u_{n, m}^{(2)}$ is the minimal nonnegative solution to the inhomogeneous semilinear equation

$$
\begin{aligned}
& \frac{\partial u_{n, m}^{(2)}}{\partial t}=L u_{n, m}^{(2)}+\beta_{2} u_{n, m}^{(2)}-\alpha\left(u_{n, m}^{(2)}\right)^{p}-\psi_{n, m} \quad \text { on } B_{2 m} \times(0, \infty) ; \\
& u_{n, m}^{(2)}(x, 0)=0, \quad x \in B_{2 m} ; \\
& u_{n, m}^{(2)} \geqslant 0,
\end{aligned}
$$

and $0 \leqslant \psi_{n, m} \leqslant n$ is a function on $\mathbb{R}^{d}$ vanishing on $B_{m}$ and equal to $n$ on $\left\{x \in \mathbb{R}^{d} \mid \operatorname{dist}\left(x, B_{m}\right) \geqslant 1 / n\right\}$. One has $u_{m}^{(2)}=\lim _{n \rightarrow \infty} u_{n, m}^{(2)}$. See the proof of [10, Theorem 3.4] for more elaboration; see also [11].)

By (3.3) and the definition of $v_{m}$, we have

$$
\lim _{m \rightarrow \infty} v_{m}=0
$$

thus, by (3.7) we have

$$
\lim _{m \rightarrow \infty} u_{m}^{(2)}=0,
$$

which contradicts (3.4). 


\section{Proof of Theorem 4}

Proof of part (1). We will show that uniqueness does not hold for (1.8). Then by Theorem EP1, the compact support property does not hold. By the comparison result stated after the proof of Theorem EP3, we may assume that $\beta=\left(\beta_{0}+\kappa\right) /|x|^{2}$. Since the problem is now radially symmetric, it suffices to show that there exists a nontrivial solution to the radially symmetric equation

$$
\begin{aligned}
& u_{t}=\frac{1}{2} u_{r r}+\frac{d-1}{2 r} u_{r}+\frac{\beta_{0}+k}{r^{2}} u-u^{p}, \quad r \in(0, \infty), t>0 \\
& u(r, 0)=0, \quad r \in(0, \infty) \\
& u \geqslant 0, \quad r \in(0, \infty), t \geqslant 0 .
\end{aligned}
$$

The function $W(x)=\kappa^{1 /(p-1)} r^{-2 /(p-1)}$ is a positive, stationary solution of the parabolic equation $u_{t}=\frac{1}{2} u_{r r}+$ $\frac{d-1}{2 r} u_{r}+\frac{\beta_{0}+\kappa}{r^{2}} u-u^{p}$ in $(0, \infty)$. By [15, Theorem 2-ii], the fact that there exists a nontrivial positive, stationary solution guarantees that uniqueness does not hold for the corresponding parabolic equation with initial data 0 ; that is, uniqueness does not hold for (4.1). Actually, the result in [15] is for equations with domain $\mathbb{R}^{d}, d \geqslant 1$, whereas the domain here is $(0, \infty)$. One can check that the proof also holds in a half space, but more simply, one can make the change of variables $z=\frac{1}{x}-x$, which converts the problem to all of $R$.

Proof of part (2). We will show that uniqueness holds for (1.8). Then by Theorem EP1, the compact support property holds. For $\epsilon$ and $R$ satisfying $0<\epsilon<1$ and $R>1$, and for some $l \in(0,1]$, define

$$
\phi_{R, \epsilon}(x)=((|x|-\epsilon)(R-|x|))^{-2 /(p-1)}(1+|x|)^{2 /(p-1)}\left(1+\frac{\epsilon^{l}}{|x|^{l}} R^{2 /(p-1)}\right) .
$$

Also, for $R$ and $\epsilon$ as above, and some $\gamma>0$, define

$$
\psi_{R, \epsilon}(x, t)=\phi_{R, \epsilon}(x) \exp (\gamma(t+1)) .
$$

Note that $\psi_{R, \epsilon}(x, 0)>0$, for $|x| \in(\epsilon, R)$, and $\psi_{R, \epsilon}(x, t)=\infty$, for $|x|=\epsilon$ and $|x|=R$. We will show that for all sufficiently large $R$ and all sufficiently small $\epsilon$, and for $\gamma$ sufficiently large and $l$ sufficiently small, independent of those $R$ and $\epsilon$, one has

$$
\frac{1}{2} \Delta \psi_{R, \epsilon}+\beta \psi_{R, \epsilon}-\psi_{R, \epsilon}^{p}-\left(\psi_{R, \epsilon}\right)_{t} \leqslant 0, \quad \text { for } \epsilon<|x|<R \text { and } t>0 .
$$

It then follows from the maximum principle for semi-linear equations [11, Proposition 1] that every solution $u(x, t)$ to (1.8) satisfies

$$
u(x, t) \leqslant \psi_{R, \epsilon}(x, t), \quad \text { for } \epsilon<|x|<R \text { and } t \in[0, \infty) .
$$

Substituting (4.2) and (4.3) in (4.5), letting $\epsilon \rightarrow 0$, and then letting $R \rightarrow \infty$, we conclude that $u(x, t) \equiv 0$. Thus, it remains to show (4.4).

From now on we will use radial coordinates, writing $\phi(r)$ for $\phi(x)$ with $|x|=r$ and similarly for $\psi$. We have

$$
\begin{aligned}
\exp ( & -\gamma(t+1))\left(\psi_{R, \epsilon}\right)_{r} \\
= & -\left(\frac{2}{p-1}\right)((r-\epsilon)(R-r))^{-2 /(p-1)-1}(R+\epsilon-2 r)(1+r)^{2 /(p-1)}\left(1+\frac{\epsilon^{l}}{r^{l}} R^{2 /(p-1)}\right) \\
& +\left(\frac{2}{p-1}\right)((r-\epsilon)(R-r))^{-2 /(p-1)}(1+r)^{2 /(p-1)-1}\left(1+\frac{\epsilon^{l}}{r^{l}} R^{2 /(p-1)}\right) \\
& -l((r-\epsilon)(R-r))^{-2 /(p-1)}(1+r)^{2 /(p-1)} \frac{\epsilon^{l}}{r^{l+1}} R^{2 /(p-1)},
\end{aligned}
$$


and

$$
\begin{aligned}
\exp ( & -\gamma(t+1))((r-\epsilon)(R-r))^{-2 /(p-1)-2}\left(\frac{1}{2} \psi_{R, \epsilon}\right)_{r r} \\
= & \left(\frac{1}{p-1}\right)(2 /(p-1)+1)(R+\epsilon-2 r)^{2}(1+r)^{2 /(p-1)}\left(1+\frac{\epsilon^{l}}{r^{l}} R^{2 /(p-1)}\right) \\
& +\left(\frac{2}{p-1}\right)(r-\epsilon)(R-r)(1+r)^{2 /(p-1)}\left(1+\frac{\epsilon^{l}}{r^{l}} R^{2 /(p-1)}\right) \\
& -\left(\frac{2}{p-1}\right)^{2}(r-\epsilon)(R-r)(R+\epsilon-2 r)(1+r)^{2 /(p-1)-1}\left(1+\frac{\epsilon^{l}}{r^{l}} R^{2 /(p-1)}\right) \\
& +l\left(\frac{2}{p-1}\right)(r-\epsilon)(R-r)(R+\epsilon-2 r)(1+r)^{2 /(p-1)} \frac{\epsilon^{l}}{r^{l+1}} R^{2 /(p-1)} \\
& +\left(\frac{1}{p-1}\right)\left(\frac{2}{p-1}-1\right)((r-\epsilon)(R-r))^{2}(1+r)^{2 /(p-1)-2}\left(1+\frac{\epsilon^{l}}{r^{l}} R^{2 /(p-1)}\right) \\
& -l\left(\frac{2}{p-1}\right)((r-\epsilon)(R-r))^{2}(1+r)^{2 /(p-1)-1} \frac{\epsilon^{l}}{r^{l+1}} R^{2 /(p-1)} \\
& +\frac{l(l+1)}{2}((r-\epsilon)(R-r))^{2}(1+r)^{2 /(p-1)} \frac{\epsilon}{r^{l+2}} R^{2 /(p-1)} .
\end{aligned}
$$

Using (4.3), (4.6) and the fact that $\frac{2}{p-1}+2=\frac{2 p}{p-1}$, we have

$$
\begin{aligned}
\exp ( & -\gamma(t+1))((r-\epsilon)(R-r))^{-2 /(p-1)-2}\left(\frac{d-1}{2 r}\left(\psi_{R, \epsilon}\right)_{r}+\beta \psi_{R, \epsilon}-\psi_{R, \epsilon}^{p}-\left(\psi_{R, \epsilon}\right)_{t}\right) \\
= & -\left(\frac{2}{p-1}\right) \frac{d-1}{2 r}(r-\epsilon)(R-r)(R+\epsilon-2 r)(1+r)^{2 /(p-1)}\left(1+\frac{\epsilon^{l}}{r^{l}} R^{2 /(p-1)}\right) \\
& +\left(\frac{2}{p-1}\right) \frac{d-1}{2 r}((r-\epsilon)(R-r))^{2}(1+r)^{2 /(p-1)-1}\left(1+\frac{\epsilon^{l}}{r^{l}} R^{2 /(p-1)}\right) \\
& -l \frac{d-1}{2 r}((r-\epsilon)(R-r))^{2}(1+r)^{2 /(p-1)} \frac{\epsilon^{l}}{r^{l+1}} R^{2 /(p-1)} \\
& +(\beta-\gamma)((r-\epsilon)(R-r))^{2}(1+r)^{2 /(p-1)}\left(1+\frac{\epsilon^{l}}{r^{l}} R^{2 /(p-1)}\right) \\
& -(1+r)^{2 p /(p-1)}\left(1+\frac{\epsilon^{l}}{r^{l}} R^{2 /(p-1)}\right)^{p} \exp ((p-1) \gamma(t+1)) .
\end{aligned}
$$

We will show that for all sufficiently large $R$ and sufficiently small $\epsilon$, and for sufficiently large $\gamma$ and sufficiently small $l$, independent of those $R$ and $\epsilon$, the sum of the right-hand sides of (4.7) and (4.8) is non-positive. This will then prove (4.4).

We will denote the seven terms on the right-hand side of (4.7) by $J_{1}-J_{7}$, and the five terms on the right-hand side of (4.8) by $I_{1}-I_{5}$. Note that the terms that are positive are $J_{1}, J_{2}, J_{4}, J_{5}, J_{7}$ and $I_{2}$. (Since $\beta$ is bounded from above, $I_{4}$ is negative for $\gamma$ sufficiently large.) In what follows, $M$ will denote a positive number that can be made as large as one desires by choosing $\gamma$ sufficiently large. Consider first those $r$ satisfying $r \geqslant c R$, where $c$ is a fixed positive number. For $r$ in this range, we have $\left|I_{5}\right| \geqslant M R^{2 /(p-1)+2}\left(1+\epsilon^{l} R^{2 /(p-1)-l}\right)$. It is easy to see that for $M$ sufficiently large, $\left|I_{5}\right|$ dominates each of the positive terms, uniformly over large $R$ and small $\epsilon$, and thus (since $M$ can be made arbitrarily large) also the sum of all of the positive terms. Now consider those $r$ for which $\delta_{0} \leqslant r \leqslant C$, for some constants $0<\delta_{0}<C$. For $r$ in this range, we have $\left|I_{4}\right| \geqslant M R^{2}\left(1+\epsilon^{l} R^{2 /(p-1)}\right)$, and it is easy to see that for $M$ sufficiently large, $\left|I_{4}\right|$ dominates each of the positive terms, uniformly over large $R$ and small $\epsilon$, and thus, also the sum of all of the positive terms. One can also show that the transition from $r$ of order unity to $r$ of order $R$ causes no problem. Thus, we conclude that for any fixed $\delta_{0}>0$, for all $l \in(0,1]$ and $\gamma$ sufficiently large, the sum of the right-hand sides of (4.7) and (4.8) is negative for all large $R$ and small $\epsilon$. 
We now turn to the case that $\epsilon \leqslant r \leqslant \delta_{0}$. (Note that at $r=\epsilon$, all the terms vanish except $J_{1}$ and $I_{5}$. Using the fact that $\frac{2}{p-1}+2=\frac{2 p}{p-1}$, it is easy to see that for sufficiently large $\gamma,\left|I_{5}(\epsilon)\right|$ dominates $J_{1}(\epsilon)$, uniformly over all large $R$ and small $\epsilon$. However, when $r$ is small, but on an order larger than $\epsilon$, the analysis becomes a lot more involved.) In the sequel, whenever we say that a condition holds for $\gamma$ or $M$ sufficiently large, or for $l$ sufficiently small, we mean that it holds independent of $R$ and $\epsilon$.

Clearly, $J_{5} \leqslant\left|I_{4}\right|$ if $\gamma$ is sufficiently large. We now show that for $\gamma$ sufficiently large, $J_{2} \leqslant\left|I_{4}\right|+\left|I_{5}\right|$, for $\epsilon \leqslant r \leqslant \delta_{0}$. (We are reusing $\left|I_{4}\right|$ here. Later we will reuse $\left|I_{5}\right|$. This is permissible because $\gamma$ can be chosen as large as we like.) To show this inequality, it suffices to show that for $M$ sufficiently large,

$$
(r-\epsilon) R \leqslant M(r-\epsilon)^{2} R^{2}+M\left(1+\frac{\epsilon^{l}}{r^{l+1}} R^{2 /(p-1)}\right)^{p-1}, \quad \text { for } r \in\left[\epsilon, \delta_{0}\right] .
$$

A trivial calculation shows that the left-hand side of (4.9) is less than the first term on the right-hand side if $r \geqslant \epsilon+\frac{1}{R M}$. If $r \in\left[\epsilon, \epsilon+\frac{1}{R M}\right]$, then the left-hand side of (4.9) is less than or equal to $\frac{1}{M}$ while the second term on the right-hand side is greater than $M$. We conclude that (4.9) holds with $M \geqslant 1$.

Since $I_{2}$ has the factor $(r-\epsilon)^{2}$, while $I_{1}$ has the factor $(r-\epsilon)$, and since $\frac{R-r}{R+\epsilon-2 r}$ can be made arbitrarily close to one by choosing $R$ sufficiently large, it follows that for any $\eta>0$, we can guarantee that $I_{2} \leqslant \eta\left|I_{1}\right|$, for $r \in\left[\epsilon, \delta_{0}\right]$, if we choose $\delta_{0}$ sufficiently small. Note that $J_{4} \leqslant \frac{2 l}{d-1}\left|I_{1}\right|$. Thus, given any $\zeta>0$, if we choose $\delta_{0}$ and $l$ sufficiently small, we will have $I_{2}+J_{4} \leqslant \zeta\left|I_{1}\right|$.

To complete the proof, we will show that

$$
J_{1}+J_{7}+(1-\zeta) I_{1}+I_{4}+I_{5} \leqslant 0, \quad \text { for } r \in\left[\epsilon, \delta_{0}\right],
$$

for sufficiently large $\gamma$ and sufficiently small $l$ and $\delta_{0}$, uniformly over large $R$ and small $\epsilon$. By the assumption on $\beta$, there exists an $\eta_{0}>0$ such that if $\delta_{0}$ is chosen sufficiently small, then

$$
I_{4} \leqslant\left(\beta_{0}-\eta_{0}\right)\left(1-\frac{\epsilon}{r}\right)^{2}(R-r)^{2}(1+r)^{2 /(p-1)}\left(1+\frac{\epsilon^{l}}{r^{l}} R^{2 /(p-1)}\right), \quad \text { for } r \in\left[\epsilon, \delta_{0}\right],
$$

where $\beta_{0}$ is as in the statement of the theorem. Since $\frac{R+\epsilon-2 r}{R-r}$ can be made arbitrarily close to one by choosing $R$ sufficiently large, we also have

$$
I_{1} \leqslant-\left(\frac{d-1}{p-1}-\frac{\eta_{0}}{2}\right)\left(1-\frac{\epsilon}{r}\right)(R-r)^{2}(1+r)^{2 /(p-1)}\left(1+\frac{\epsilon^{l}}{r^{l}} R^{2 /(p-1)}\right), \quad \text { for } r \in\left[\epsilon, \delta_{0}\right] .
$$

Since

$$
\begin{aligned}
J_{7} & =\frac{l(l+1)}{2}((r-\epsilon)(R-r))^{2}(1+r)^{2 /(p-1)} \frac{\epsilon^{l}}{r^{l+2}} R^{2 /(p-1)} \\
& \leqslant \frac{l(l+1)}{2}(R-r)^{2}(1+r)^{2 /(p-1)} \frac{\epsilon^{l}}{r^{l}} R^{2 /(p-1)},
\end{aligned}
$$

and since $\frac{R-r}{R+\epsilon-2 r}$ can be made arbitrarily close to one by choosing $R$ sufficiently large, it follows that for any $\tau>0$, we can choose $l$ sufficiently small so that

$$
J_{7} \leqslant \tau J_{1}, \quad r \in\left[\epsilon, \delta_{0}\right],
$$

uniformly over large $R$ and small $\epsilon$. Using (4.11)-(4.13) along with the fact that

$$
J_{1} \leqslant \frac{p+1}{(p-1)^{2}}(R-r)^{2}(1+r)^{2 /(p-1)}\left(1+\frac{\epsilon^{l}}{r^{l}} R^{2 /(p-1)}\right)
$$

and that $\beta_{0}=\frac{d(p-1)-2 p}{(p-1)^{2}}$, it follows that

$$
\begin{aligned}
J_{1}+J_{7}+(1-\zeta) I_{1}+I_{4} \leqslant & \left(\frac{\tau(p+1)+\zeta(d-1)(p-1)}{(p-1)^{2}}-\eta_{0}\left(\frac{1}{2}+\frac{\zeta}{2}\right)+C \frac{\epsilon}{r}\right) \\
& \times(R-r)^{2}(1+r)^{2 /(p-1)}\left(1+\frac{\epsilon^{l}}{r^{l}} R^{2 /(p-1)}\right), \quad \text { for } r \in\left[\epsilon, \delta_{0}\right],
\end{aligned}
$$


for some $C>0$, uniformly over large $R$ and small $\epsilon$. By picking $\tau$ and $\zeta$ sufficiently small, we have

$$
\frac{\tau(p+1)+\zeta(d-1)(p-1)}{(p-1)^{2}}-\eta_{0}\left(\frac{1}{2}+\frac{\zeta}{2}\right)<0 .
$$

Thus, in order to show (4.10), it suffices to show that

$$
\frac{\epsilon}{r} R^{2} \leqslant M\left(1+\frac{\epsilon^{l}}{r^{l}} R^{2 /(p-1)}\right)^{p-1}, \quad r \in\left[\epsilon, \delta_{0}\right],
$$

for sufficiently large $M$. But since $l(p-1) \leqslant 1$, the right-hand side of (4.15) is greater or equal to $M \frac{\epsilon}{r} R^{2}$.

\section{Acknowledgements}

\section{R. P. thanks Robert Adler for a helpful conversation.}

\section{References}

[1] P. Baras, M. Pierre, Problèmes paraboliques semi-linéaires avec données mesures, Appl. Anal. 18 (1984) 111-149.

[2] H. Brezis, L. Veron, Removable singularities for some nonlinear elliptic equations, Arch. Rational Mech. Anal. 75 (1980) 1-6.

[3] D.A. Dawson, Measure-valued Markov processes, in: École d'Été de Probabilités de Saint-Flour XXI-1991, in: Lecture Notes in Math., vol. 1541, Springer, Berlin, 1993, pp. 1-260.

[4] D.A. Dawson, I. Iscoe, E.A. Perkins, Super-Brownian motion: path properties and hitting probabilities, Probab. Theory Related Fields 83 (1989) 135-205.

[5] R.D. DeBlassie, On hitting single points by a multidimensional diffusion, Stochastics Stochastics Rep. 65 (1998) 1-11.

[6] E.B. Dynkin, A probabilistic approach to one class of nonlinear differential equations, Probab. Theory Related Fields 89 (1991) $89-115$.

[7] E.B. Dynkin, Superprocesses and partial differential equations, Ann. Probab. 21 (1993) 1185-1262.

[8] S. Dynkin, E.B. Kuznetsov, Superdiffusions and removable singularities for quasilinear partial differential equations, Comm. Pure Appl. Math. 49 (1996) 125-176.

[9] J. Engländer, Criteria for the existence of positive solutions to the equation $\rho \Delta u=u^{2}$ in $\mathbb{R}^{d}$ for all $d \geqslant 1-$ a new probabilistic approach, Positivity 4 (2000) 327-337.

[10] J. Engländer, R. Pinsky, On the construction and support properties of measure-valued diffusions on $D \subset \mathbb{R}^{d}$ with spatially dependent branching, Ann. Probab. 27 (1999) 684-730.

[11] J. Englan̈der, R. Pinsky, Uniqueness/nonuniqueness for nonnegative solutions of second-order parabolic equations of the form $u_{t}=L u+$ $V u-\gamma u^{p}$ in $\mathbb{R}^{n}$, J. Differential Equations 192 (2003) 396-428.

[12] K. Fleischmann, A. Sturm, A super-stable motion with infinite mean branching, Ann. Inst. H. Poincaré Probab. Statist. 40 (2004) $513-537$.

[13] A. Pazy, Semigroups of Linear Operators and Applications to Partial Differential Equations, Springer-Verlag, New York, 1983.

[14] R. Pinsky, Positive Harmonic Functions and Diffusion, Cambridge University Press, 1995.

[15] R. Pinsky, Positive solutions of reaction diffusion equations with super-linear absorption: universal bounds, uniqueness for the Cauchy problem, boundedness of stationary solutions, J. Differential Equations, in press.

[16] Y. Ren, Support properties of super-Brownian motions with spatially dependent branching rate, Stochastic Process. Appl. 110 (2004) 19-44.

[17] L. Veron, Singular solutions of some nonlinear elliptic equations, Nonlinear Anal. 5 (1981) 225-242. 